\title{
Artificial intelligence- the new suggestion for biomedicine, dentistry and healthcare
}

\author{
M. Enchev, J. Kostadinov, S. Peev, D. Kostadinova, S. Toncheva, M. Stoykov, \\ G. Georgieva, M. Milkov \\ Department of Dental Materials Science and Propaedeutics in Prosthetic Dentistry, \\ Faculty of Dental Medicine, Medical University - Varna
}

\begin{abstract}
The development of technologies based on Artificial Intelligence (AI) and their application in medicine is growing rapidly. Innovations in digital technology, telemedicine, 5G technology and artificial intelligence (AI) create new opportunities for the development of the healthcare system. The aim of the present study is to explore the possibilities for the application of artificial intelligence in biomedicine, dentistry, healthcare and healthcare. In recent years there have been many major innovations, including the introduction of many new information and communication technologies. Digital innovations, including the further inclusion of telemedicine, the development of 5th generation wireless networks (5G) and artificial intelligence (AI) approaches, create an exceptional ecosystem for new health opportunities. The digital health sector creates a favorable environment for the provision of health services at a very high level. Keywords: artificial intelligence, $5 \mathrm{G}$ technology, telemedicine, digital technologies, dental health, biomedicine, health care
\end{abstract}

\section{Introduction}

Artificial intelligence is not a cybernetic nous, but a system of algorithms that process vast amounts of data and learns on the basis of machine learning. In the future, artificial intelligence will definitely free us from performing routine tasks in many areas of life. One of the most promising directions for its use is in the field of medicine and healthcare. The development of technologies based on Artificial Intelligence and their application in medicine is growing at a very fast pace. The Artificial Intelligence application in healthcare serves for treatment algorithm creations in regard to different patient characteristic and particular treatment models according to each patient individual values. The most complex forms machine-based learning include many levels of functions and variables which predict the end-results

\section{Aim}

The aim of the present study is to investigate the possibilities of application of artificial intelligence in biomedicine, dentistry, healthcare and healthcare.

\section{Material and methods}

For the period October 2020- October 2021 in the available database (PubMed, BioMedCentral, ScienceDirect, Scopus, Web of Science) a systematic analysis of scientific publications examining the application of artificial intelligence in biomedicine, dentistry, healthcare and healthcare was conducted. 


\section{Results and discussion}

There have been many major innovations in recent years, including the introduction of many new information and communication technologies. Digital innovations, including the further inclusion of telemedicine, the development of 5th generation wireless networks (5G) and artificial intelligence (AI) approaches, create an exceptional ecosystem for new healthcare opportunities. The digital healthcare sector creates a favorable environment for the provision of health services at a very high level. Artificial Intelligence accomplishes healthcare tasks which could aid and assist healthcare specialist. During the past years many ethical questions has been discussed about the application artificial intelligence in the healthcare sector.

In the field of medicine, the term "artificial intelligence" is more optimistic and confident that we will witness a revolution that will help not only doctors but patients as well. One of the possibilities for this lies in the voice assistants. The British market analysis company Juniper Research predicts that the use of voice assistants will triple in the next few years. Thus, in 2023, there will be 8 billion digital voice assistants in use, compared to 2.5 billion by the end of 2018. For patients, this could mean a lot.

This type of intelligent system can provide 24-hour care for a wide range of people who may need help. People with chronic illnesses who have seizures, people with disabilities and those who live far from medical care are just some of those who would benefit the most from virtual assistants. Beyond popular solutions such as Amazon's Alexa, Alphabet's Google Assistant, and Apple's Siri, there are similar assistants focused specifically on medicine.

The program of the software company Nuance Communications, which was created specifically for medical purposes and is called Dragon Medical Virtual Assistant. It is part of the existing voice-totext software used for clinical documentation.(3)

According to the company's website, Dragon Medical is used by more than 500,000 doctors to create and communicate the cases of more than 300 million patient cases a year. The assistant himself is designed so that artificial intelligence understands and recognizes medical terminology and systematizes information in a way of doctors need. (3) Artificial intelligence can help us to better process and analyze medical data, as well as to develop drug molecules, to provide care, examina- tions and prevention at a distance.

\section{What is artificial intelligence used for in medicine?}

Since the early 1970s diagnosis and treatment of disease has been a focus of AI. (8)

\section{Diagnosis}

Making the right diagnosis is the key to taking the right treatment and cure of patient. In recent years, artificial intelligence has developed in this direction - to be able to adequately replace a diagnostician and make a correct diagnosis based on many previous ones. Examples: recognition of cancers based on computed tomography; recognition of risk of heart diseases on the basis of electrocardiograms and cardiac MRI;(6) classification of skin lesions; identification of diabetic retinopathy in eye images. Development of new drugs: Artificial intelligence is used in all four phases of drug development. It is used to identify the right disease proteins that the drug affects, and then to find the right compounds that would interact with those proteins. Algorithms go through millions of possible options to get to the right ingredients that would lead to minimal side effects. They also help to identify the right biomarkers, which can monitor the effectiveness of the drug, the development of the disease and make the right choices before the release of the drug for mass use.

\section{Personalized treatment}

Different patients respond differently to certain drugs, and artificial intelligence greatly helps in choosing the right drugs for specific patients. The system compares the symptoms and reactions of different patients to a treatment and helps doctors choose the right and safe treatment. Genetic editing: Genetic editing made possible by the CRISPR (Clustered Regularly Interspaced Short Palindromic Repeats) system, which allows specific modification of certain genes, such as the elimination of those that lead to autoimmune diseases (see video at the end of the article). Algorithms in this case can help identify unwanted side effects and develop technology in the future. Patient data processing: Each medical record, examination report and medical history contains information that allows diagnosis and treatment to be prescribed. Unfortunately, even experienced doctors do not always see the full 
picture of the disease, because the data in medical documents are not well structured, and the history of the disease can be quite extensive. In some cases, work efficiency is affected by factors such as fatigue, and in others, such as insufficient knowledge in a specific narrow field. In order for a disease, such as cancer, to be effectively treated, some non-obvious symptoms need to be recognized. According to Google, one in ten patients suffers from misinterpretation of medical information.

Artificial intelligence can solve this problem. (5) Google (Deepmind Health) (7) and IBM (Watson Health) (9) have such developments for patient assessment and preliminary diagnosis. Google products now operate in several medical centers in the United Kingdom and the United States. One of them is the Moorfields Ophthalmology Clinic, where Google Deepmind Health analyzes the available information about the patient's symptoms and gives a list of recommendations. The doctor, using the advice of his "digital assistant", prescribes a course of treatment.

IBM Watson Health also helps with diagnoses. It recognizes venous thrombosis, cardiomyopathy and heart attacks. There has been a case where Dr. Watson diagnosed a 60-year-old patient with a rare form of leukemia where the woman was initially misdiagnosed. In this case, the system had to "study" 20 million scientific articles in only 10 minutes. According to Frost \& Sullivan, artificial intelligence technologies increase the accuracy of diagnoses by $30-40 \%$, while reducing the cost of medical care by half.

For example, The Johns Hopkins Hospital claims that the intellectual system, developed in collaboration with GE Healthcare Partners, can speed up the process of prescribing appropriate treatment by $30 \%$.

Artificial intelligence is also used in cochlear implant telemedicine. (12)
The introduction of Artificial Intelligence in dental medicine will make it even more accurate and will increase the quality and speed of services provided to patients.(11)

During the past year there has been a growing interest in the Artificial Intelligence technique utilization in biomedical engineering and processing information, ranging from knowledge-based reasoning for disease classification to learning and discovering novel biomedical knowledge for disease treatment.(10)

\section{Conclusion}

In the future, applications using artificial intelligence are expected to gain access to large databases with disease histories, genetic information and medical literature. In this way, they will be able to give their recommendations and guidelines for proper treatment much more precisely. The use of artificial intelligence to search for the optimal method of treatment is necessary because the same treatment approach does not always help patients diagnosed with the same disease equally well.

The applied digital technologies will increase the quality of the offered services in the field of healthcare. Artificial intelligence is a new challenge in the field of medicine that will help healthcare professionals on the one hand and improve the lives of patients on the other.

Teleconsultation, Precision medicine and genomics are just some of the contemporary fields that rapidly evolve around the utilization of the Artificial Intelligence application in the clinical practice. The ethical side plays an important role in the field of artificial intelligence by virtue of financial issues that occur while trying to be transparent and accurate in developing new solutions to improve contemporary healthcare

\section{References}

1. Davenport T, Kalakota R. The potential for artificial intelligence in healthcare. Future Healthc J 2019; 6(2):94. [cited 2019 Sep 23]. Available from: http://www.ncbi.nlm.nih.gov/pubmed/31363513.

2. Lee SI, Celik S, Logsdon BA et al. A machine learning approach to integrate big data for precision medicine in acute myeloid leukemia. Nat Commun 2018; 9: 42.

3. Nuance AI-Powered Virtual Assistants for Healthcare Available from: https://www.nuance.com/healthcare/ambient-clinical-intelligence/ virtual-assistants.html

4. Oakden-Rayner L. Exploring the ChestXray14 dataset: problems. 2017 [cited 2019 Sep 23]. Available from: https://lukeoakdenrayner. wordpress.com/2017/12/18/the-chestxray14-dataset-problems/

5. Topol EJ. High-performance medicine: the convergence of human and artificial intelligence. Nat Med 2019. [cited 2019].

6. Rajpurkar P, Irvin J, Zhu K, et al. CheXNet: radiologist-level pneumonia detection on chest X-rays with deep learning. 2017; Available from: http://arxiv.org/abs/1711.05225 
7. DeepMind's health team- Google Health Available from: https://deepmind.com/blog/announcements/deepmind-health-joins-google-health

8. Bush J. How AI is taking the scut work out of health care. Harvard Business Review 2018. https://hbr.org/2018/03/how-ai-is-taking-the-scutwork-out-of-health-care.

9. Watson Health is smarter health Available from: https://www.ibm.com/watson-health

10. Peng Y, Zhang Y, Wang L. Artificial intelligence in biomedical engineering and informatics: an introduction and review. Artif IntellMed. (2010) 48: 71-3.

11. F. Schwendicke, W. Samek, J. Krois Artificial Intelligence in Dentistry: Chances and Challenges, Vol 99, Issue 7, 2020 Available from https:// journals.sagepub.com/doi/full/10.1177/0022034520915714

12. Matthias Meeuws, David Pascoal, Sebastien Janssens de Varebeke, Geert De Ceulaer, Icon \& Paul J. Govaerts Cochlear implant telemedicine: Remote fitting based on psychoacoustic self-tests and artificial intelligence Pages 260-268, 13 May 2020 Available from Cochlear Implants International An Interdisciplinary Journal for Implantable Hearing Devices Volume 21, 2020 - Issue https://www.tandfonline.com/doi/abs/10 $.1080 / 14670100.2020 .1757840$ ?journalCode=ycii20

Reviewer of the article: Prof. Dr. Hesham Negm, MD, PhD - Cairo University 